\title{
Géométries du mouvement
}

\section{Geometries of movement}

\section{Jean Letourneur ${ }^{1}$}

${ }^{1}$ Sculpteur spécialiste de la taille directe et des sciences du Chaos.

A enseigné à l'Ecole Nationale Supérieure des Arts décoratifs, à l'Ecole Nationale Supérieure des Arts Appliqués et des Métiers d'Art, à l'Ecole Nationale Supérieure d'Architecture de Paris-la-Villette.

RÉSUMÉ. Léonard de Vinci fut un précurseur dans le domaine de la mécanique des fluides, aussi bien par son approche expérimentale que par ses applications simplement artistiques. Sa démarche aura dicté la mienne.

Fidèle à cette méthode, j'ai pu investir les domaines de la Turbulence en une véritable « quête de l'unité » comparable à celle des scientifiques d'aujourd'hui lorsqu'ils tentent d'aboutir à une description cohérente des lois de la Nature.

Fondée notamment sur l'invariance d'échelle, cette quête m'aura permis de prendre place parmi les précurseurs de I' " art fractaliste ", dont Léonard aura posé les prémisses aussi bien par ses études de la croissance des plantes que par ses analyses des mouvements de l'eau.

ABSTRACT. Leonardo da Vinci was a precursor in the fluid mechanics field, thanks to his experimental approach as well as its purely artistic applications. His creative process has inspired mine.

Keeping with this spirit, I have been able to transform the turbulence areas into a genuine "unity quest", comparable to that of current scientists when they try to come up with a consistent description of the laws of Nature.

Notably based on scale invariance, this quest has allowed me to take place among the precursors of "fractal art", whose premises have been set by Leonardo not only with his studies of plant growth but also with his analyses of water movements.

MOTS-CLÉS. Art et science, turbulence, fractales, autosimilarité, chaos, discontinuités, vortex, mécanique des fluides. KEYWORDS. Art and science, turbulence, fractals, self similarity, chaos, discontinuity, vortex, fluid mechanics.

Que d'impondérables dans les circonstances qui m'ont conduites à me promener entre l'atelier paternel où je travaille encore, et les laboratoires de mécanique des fluides de l'ONÉRA ! Après bientôt quatre décennies consacrées à tenter de donner un prolongement aux dessins que Léonard a dédiés au mouvement de l'eau, demeure intact ce sentiment éprouvé grâce aux travaux d'Henri Werlé découverts en 1973, où j'entrevis en un éclair les territoires inexplorés dont l'ONÉRA me livrait les clefs.

Mais de cet immense traité d'anatomie qui soudain s'était ouvert devant moi, il me fallut dégager des articulations, des systèmes, des lois.

Lorsque science et art se confondent, ce dernier devient un instrument de la connaissance, et Léonard en avait posé les jalons par une véritable « méthode expérimentale » avant la lettre :

«On appelle connaissance mécanique celle qui est engendrée par l'expérience ; et scientifique celle qui naît et finit dans l'esprit... Mais les vraies sciences sont celles que l'expérience a fait pénétrer par les sens et qui fait faire silence aux disputeurs, et qui ne repaît pas de songe ses chercheurs, mais qui toujours, sur les principes premiers, vrais et connus, procède successivement et par de vraies séquences jusqu'à la fin... qui traitent en vérité des quantités continues et discontinues ».

En effet, de toute évidence les dessins analytiques de Léonard, fondateur de ce qu'on appelle maintenant la mécanique des fluides, furent réalisés à partir d'expérimentations concrètes -obstacles divers placés dans un courant selon des incidences variables, jets d'eau à débits contrôlés... et l'exposition du Louvre nous permet enfin d'en découvrir d'inédits [figure 1]. 


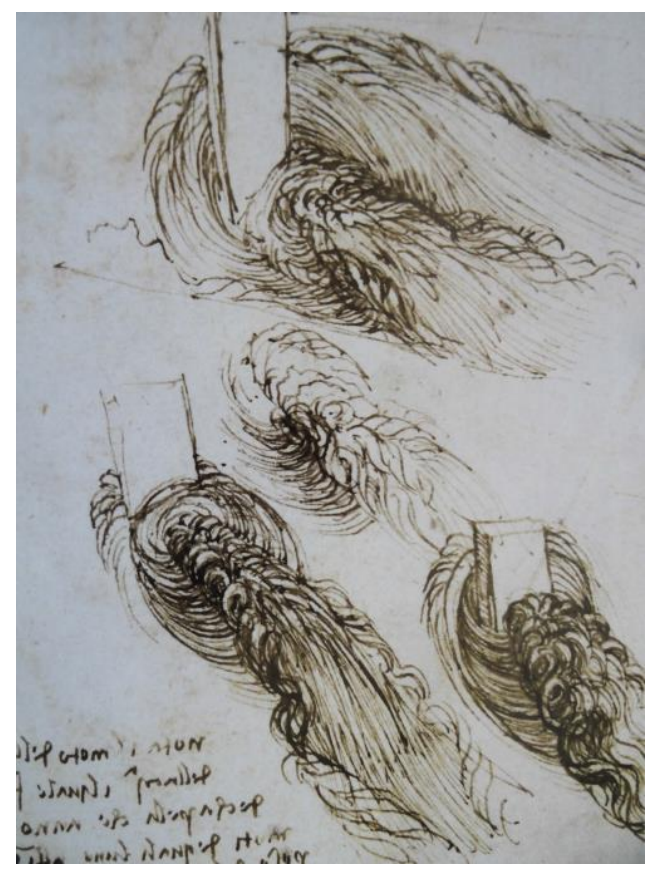

Figure 1. encre, vers 1510/1513, Windsor, $15 \times 17 \mathrm{~cm}$

Puis Léonard transposa les leçons ainsi apprises dans les registres habituels des chevelures et des drapés : le traitement des cheveux de ses personnages évoque irrésistiblement le mouvement des eaux dont il a le premier analysé les lois [figure 2]. Certains de ses drapés ne peuvent s'expliquer que par des références à l'hydrodynamique [figure 3].

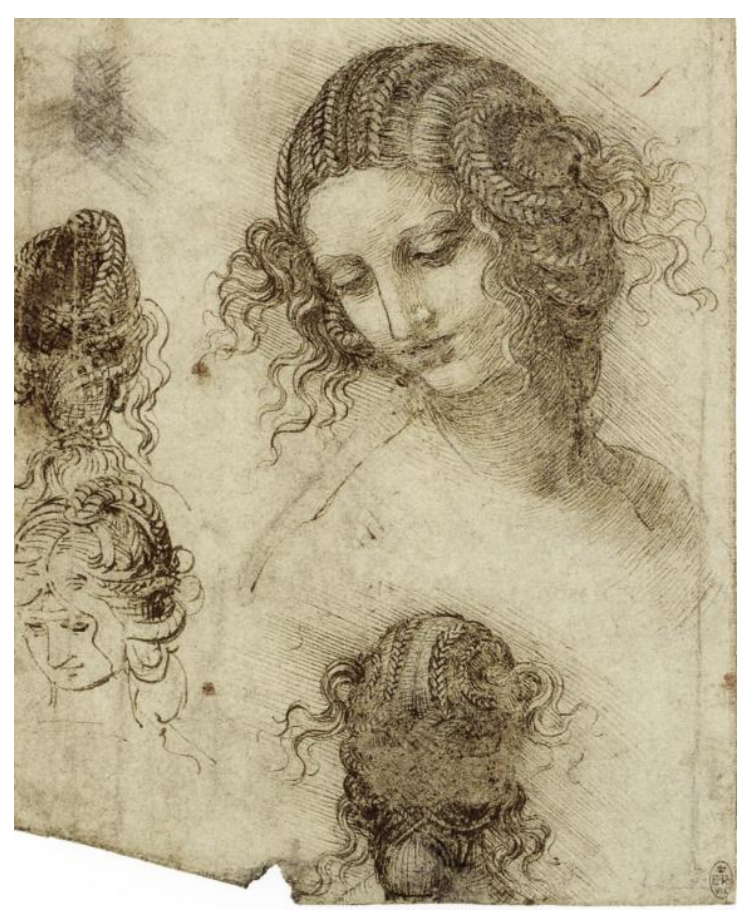

Figure 2. Léda, pierre noire et encre, vers 1505/1510, Windsor, $20 \times 16,2 \mathrm{~cm}$

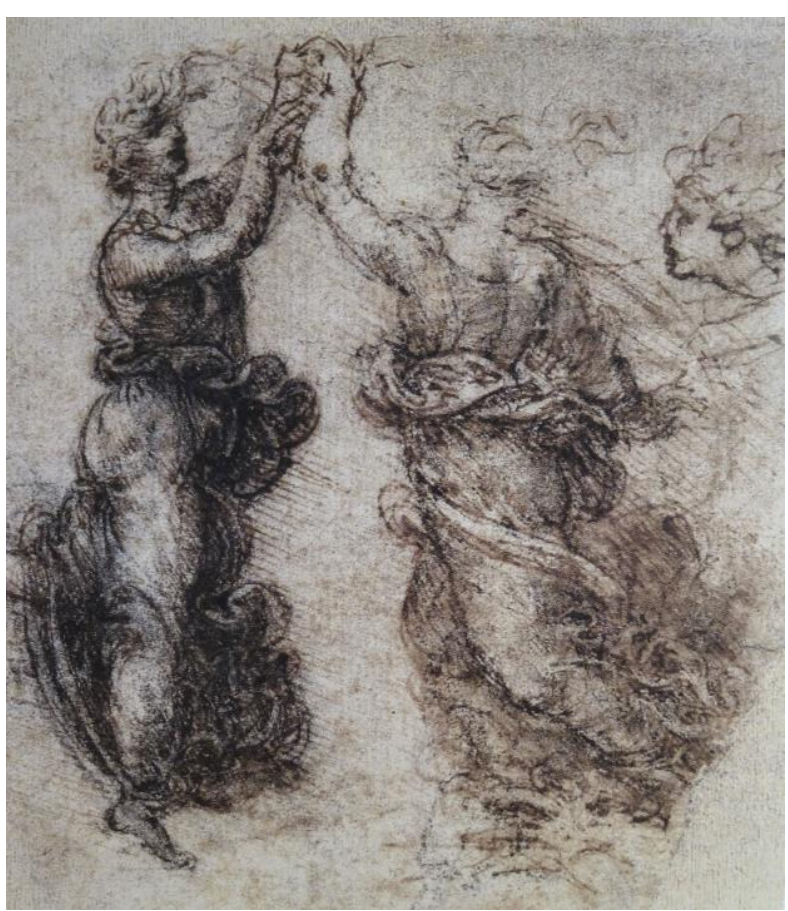

Figure 3. encre, vers 1515, Venise galerie de l'Académie, 9,6 × 14,9 cm

«Prends connaissance du mouvement de la surface de l'eau, qui se comporte comme les cheveux, lesquels ont deux directions, l'une qui suit leur poids propre, et l'autre les injonctions des boucles ; de même, l'eau décrit ses courbes tourbillonnantes, dont une partie suit le sens du courant principal, et l'autre le mouvement fortuit et induit». 
Pour livrer, à la fin de sa vie, avec la série des Déluges [figure 4], ce que l'on devrait considérer comme les premiers dessins « abstraits » de l'art occidental... que les récentes sciences du chaos ont permis de redécouvrir.

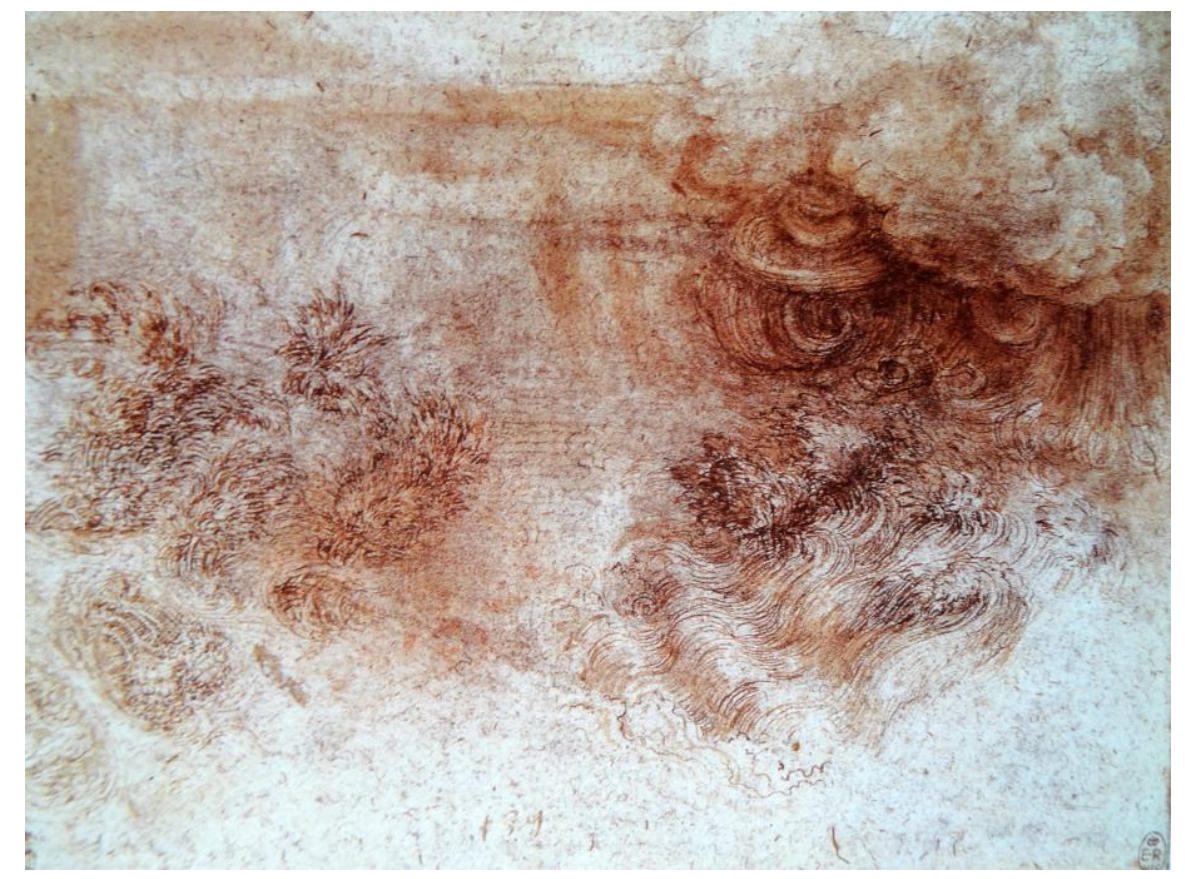

Figure 4. encre et lavis bistre, vers 1514, Windsor, 15, 7 × 20,3 cm

Mais toute médaille, aussi séduisante soit-elle, a son revers : si par ses images la science peut devenir accessible au non-scientifique -et c'est bien dans cette catégorie que se rangent les documents de Werlé- l'exemple de Léonard suppose non seulement la maîtrise du métier, mais aussi de se familiariser avec les concepts sous-tendant cette science particulière. Pour voir et comprendre ce que l'on a devant soi, il faut non seulement observer, mais aussi avoir des idées claires. Et dans ce domaine seule la science offre par ses concepts l'ouverture indispensable à qui prétend en interpréter les manifestations.

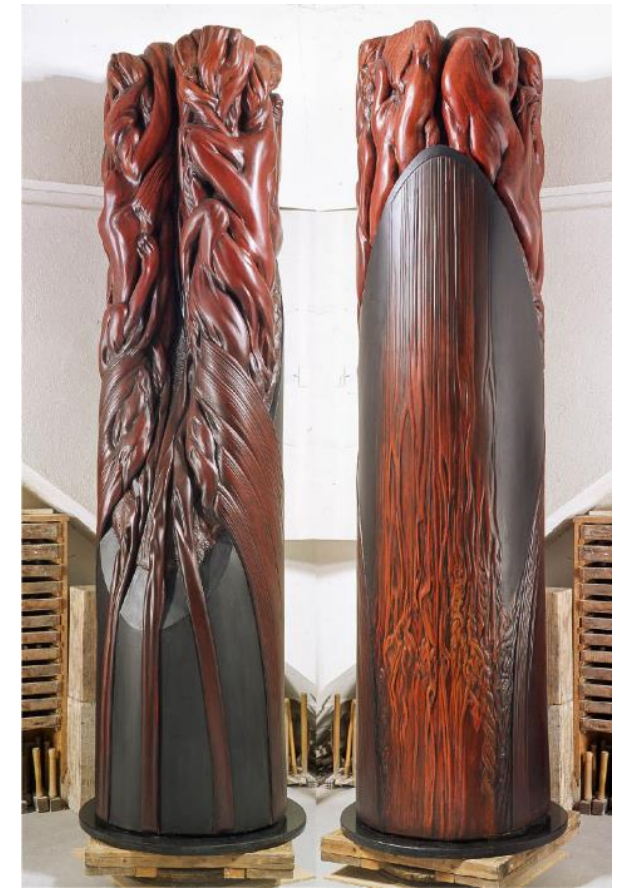

Parmi ces concepts, il me faut citer :

1) La « sensitivité aux conditions initiales » d'Henri Poincaré, dont d'infimes variations peuvent modifier l'ensemble d'un système. Alliée aux chaînes de vortex transmettant l'énergie des grandes échelles vers les petites échelles dissipatives, ce sont les clefs de la composition de Katabase [figure 5] par exemple.

Figure 5. Katabase plâtre polychrome

d'après la pierre, $300 \times 75 \times 78 \mathrm{~cm} 1991$ 
2) L'« autosimilarité » des fractales de Benoit Mandelbrot, qui relie les formes que revêt la turbulence à toutes les échelles. Ce concept se retrouve aussi bien dans mes Méharées [figure 6] que dans le traitement des formes de la partie dorsale de Citadelle [figure 7].

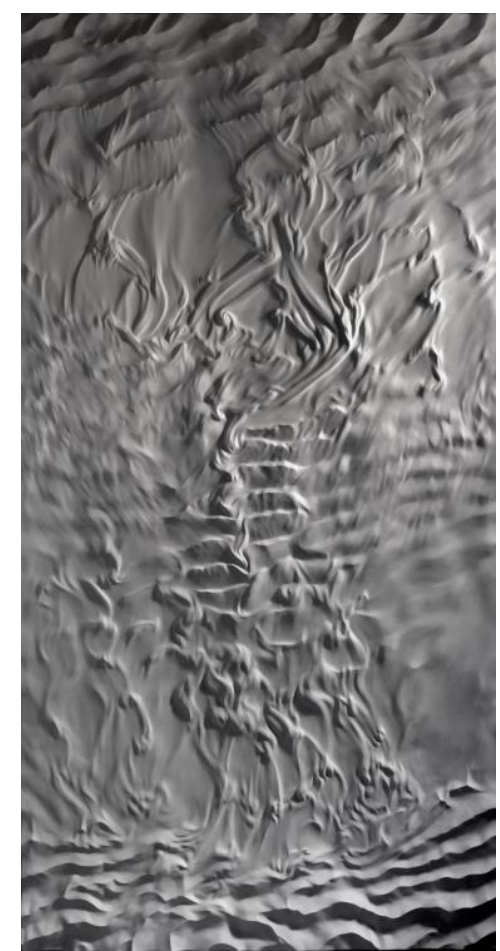

Figure 6. Marches au hasard, plâtre, $300 \times 150 \times 10 \mathrm{~cm}, 2012$

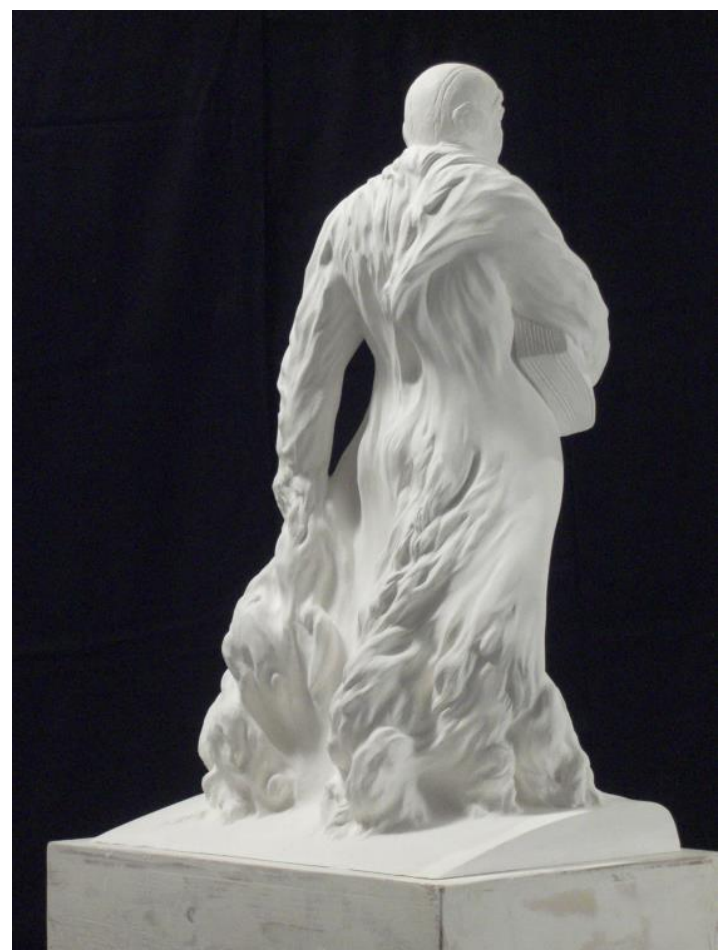

Figure 7. Citadelle, plâtre, $89 \times 57 \times 37 \mathrm{~cm}, 2010$

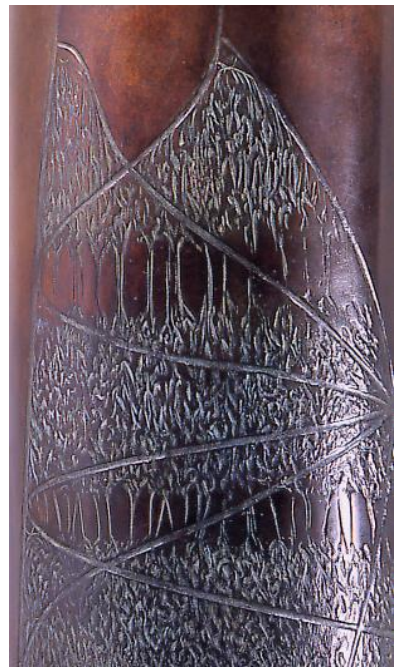

3) La « bifurcation » ou « cascades de doublement de période » de Mitchell Feigenbaum, où apparaissent paradoxalement des franges structurées entre deux phases chaotiques : ces instabilités sont bien potentiellement créatrices d'ordre (dos de Bifurcation) [figure 8]. On retrouve des franges verticales comparables dans les traces laissées par la mer sur le sable, et qui commandent la composition de Marches au Hasard [figure 6].

Figure 8. Bifurcations (détail) -

bronze- $75 \times 34,5 \times 30 \mathrm{~cm}-1995$

4) Les «corrélations à longue portée » d'Ilya Prigogine et les phénomènes d' " hystérésis » qui l'accompagnent en mécanique des fluides quand à elles me permettent de créer les mises en tension formelles équivalentes à ce que les sculpteurs faisaient par l'entremise de l'anatomie (Interférences de Chocs, voir article précédent). 
S'inspirer du caractère fractal de la turbulence, dont Mandelbrot avait souligné la correspondance, revient à composer non plus depuis l'anatomie, la géométrie, les drapés, la perspective... mais sur la base de ce hasard non dirigé offert par la Nature et dont la science contemporaine a démontré l'omniprésence depuis l'organisation du cosmos jusqu'à l'expression de certains gènes.

En revanche, il s'agit de l'investir selon le « hasard dirigé » du geste créateur !

En voici quelques exemples, et la façon dont la science a pu nourrir l'art en lui fournissant non seulement sa thématique, mais encore sa méthodologie :

KATABASE [figure 5] d'après les tourbillons contrarotatifs à l'aval du culot incliné d'un cylindre, qui avait été érigée sur la place de l'Eglise de Fontenay aux Roses, accompagnée de deux bassins alimentés par des déversoirs [figure 10].

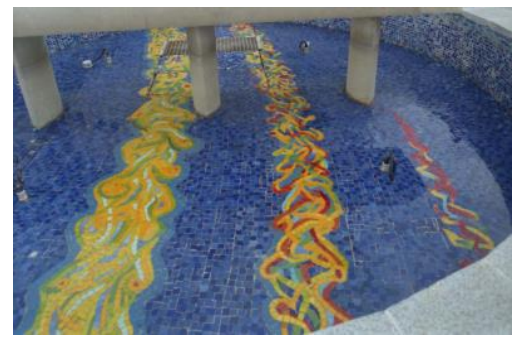

Figure 10. Mosaique, bassin de la place de l'église de Fontenay-aux-Roses (détruit)

Il s'agissait de créer un espace dynamique dans un plan vertical avec la sculpture et dans un plan horizontal avec la composition des bassins dont elle est l'axe de rotation, tout en conservant la symétrie générale de la place, la sculpture étant placée en position centrale.

La rotation virtuelle des deux déversoirs était marquée par l'axe diagonal qui les relie, par le sens alterné d'écoulement des déversoirs visant à suggérer une rotation que venait renforcer la composition de la mosaïque, conçue comme la projection horizontale des tourbillons marginaux d'un rotor d'hélicoptère au point fixe. Le Discobole [figure 11] se réfère à cette même source de turbulence, mais dans un tout autre registre.

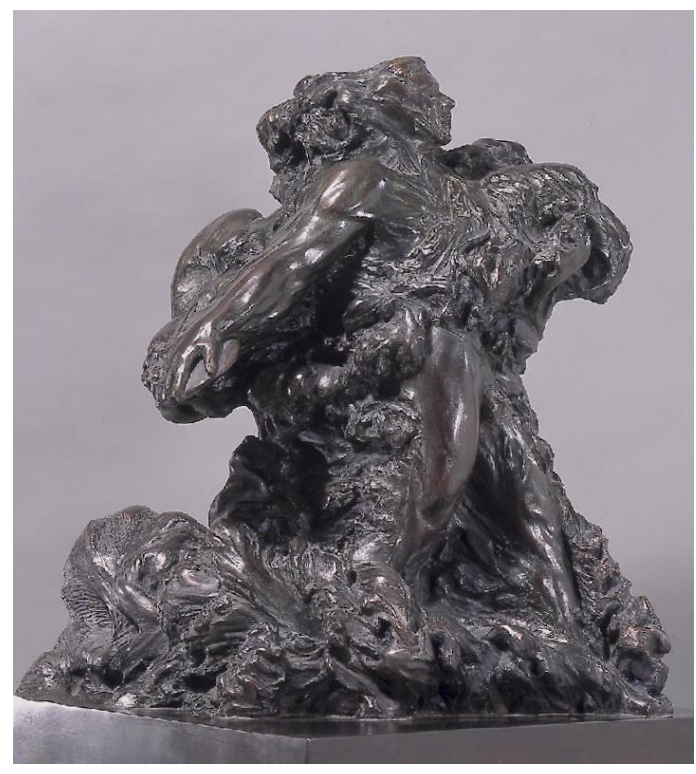

Figure 11. Le Discobole - bronze $70 \times 50$ × $50 \mathrm{~cm}-1983-2005$ 
Par analogie, les deux déversoirs sont donc comme deux pales d'hélicoptère en rotation et la lame d'eau matérialise la projection dont dépend le dessin des mosaïques établi sur trois cercles concentriques : temps $t$ calé sur l'extrémité de la lame d'eau, tourbillon marginal en formation donc dense et étroit, $t-1$ tourbillon d'un rayon légèrement inférieur, moins dense et plus dilué en largeur, et $t$-2 tourbillon largement diffusé.

Les bassins circulaires deviennent comme des oculi ouverts sur un mouvement souterrain et vivement coloré se prolongeant virtuellement sous la totalité de la place.

Le décalage des cercles dont le rayon va en décroissant à mesure que l'on remonte dans le temps trouve sa justification par le fait qu'il fallait exprimer par le dessin et par la couleur des phases antérieures qu'une superposition même partielle aurait rendues illisibles. De plus ces décalages ont été mis en évidence par les visualisations de l'ONÉRA.

La Sculpture placée sur l'axe de rotation avait été taillée directement dans un prisme octogonal dont le dessin du décaissé qui lui servait de socle gardait la mémoire.

Sa composition s'inspire essentiellement des tourbillons contrarotatifs au culot incliné d'un cylindre traités en ronde-bosse. L'angle de section est important afin de ménager au dos de ce cylindre une surface uniforme où figure, en bas-relief cette fois, une transition issue elle aussi des études de Werlé.

Les deux mouvements antagonistes, de bas en haut pour les tourbillons principaux et de haut en bas pour la transition dorsale, créent des cisaillement que j'ai traités en m'inspirant des phénomènes similaires se produisant à la surface de Jupiter et révélés par la sonde Voyager.

Nécessairement symétriques, j'ai accentué les différences de formes prises par ces tourbillons en inclinant légèrement à gauche la section plane du cylindre. La grande difficulté a été de concilier la logique de ce système dynamique telle qu'elle a été révélée par les études d'Henri Werlé et l'impression de puissance aveugle et désordonnée que donne la lecture d'un film où ces tourbillons sont révélés par les colorants. La sculpture étant une combinaison de plusieurs instants s'échelonnant dans le temps, menés par un réseau de lignes de force, il m'a fallu réunir en un ensemble cohérent des fragments analysés tout au long d'une série d'arrêts sur image.

$\mathrm{Au}$ travers de formes ayant pris corps et donc figées dans le temps, il s'agissait de retrouver une impression fondée sur des contours en mouvement. En ce sens, cette étude s'apparente à la traduction du mouvement que les sculpteurs ont des siècles durant recherché au travers de l'anatomie humaine ou des drapés.

Les «corrélations à longue portée » de Prigogine constituent le concept dominant de la partie dorsale de Katabase, régissant la totalité de la composition, avec comme toujours la sensitivité aux conditions initiales faisant voisiner des zones quasi laminaires avec d'autres où la turbulence est complètement établie. Une infime variation locale à l'origine du mouvement générant un changement de régime l'englobant tout entier, il n'y a plus de causalité avec les phénomènes non linéaires et s'en inspirer offre à la fois rigueur et liberté d'interprétation. 

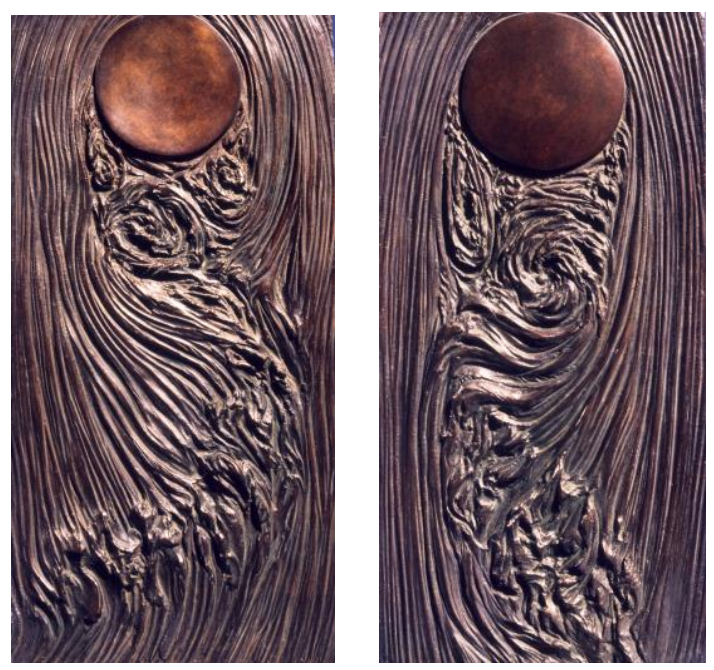

Figure 12a. L'air (Bronze, $40 \times 20 \times 2,5 \mathrm{~cm}-1992)$

Figure 12b. L'eau (Bronze, 40 × 20 × 2,5 cm-1992)
L'AIR ET L'EAU [figures 12a, 12b], deux bronzes issus des célèbres allées tourbillonnaires de Bénard-von Kàrmàn.

Par ce hasard omniprésent, nous nous trouvons toujours face à des formes restant à inventer.

Le raisonnement, bien que restant circonscrit dans un cadre parfaitement défini, devient alors d'une autre nature: non pas exclusivement rationnel et maîtrisable, mais s'apparentant à un processus dynamique toujours situé en écart à l'équilibre, faisant osciller le drapé turbulent tantôt à gauche, tantôt à droite du sillage.

La «structuration » naissant d'ondulations fluides par cascades d'échelle s'auto-génère donc par accumulation de formes en un processus expansif dont le hasard est intimement partie prenante.

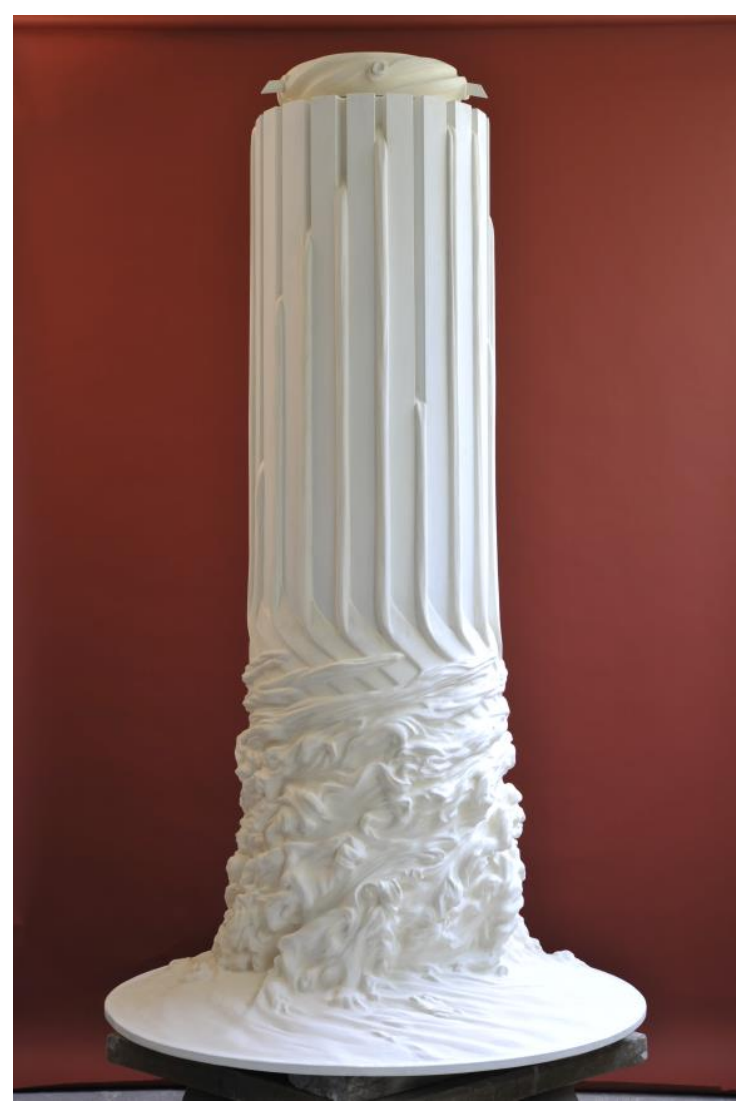

Figure 13. Fontaine Werlé - plâtre et bois, $240 \times 120 \times 120 \mathrm{~cm}-1994$
FONTAINE WERLE [figure 13]. Il s'agit d'un projet de fontaine inspiré d'un écoulement autour d'une colonne dont la partie inférieure est en rotation, cette contrainte constituant une variante sur le thème des transferts d'énergie, sujet principal de Katabase.

J'écrivais en 1983 à Henri Werlé :

«Les recherches actuelles consistant à placer des moteurs dans la sculpture pour faire pivoter des bouts de métal poli ou autres utilisations simplistes des produits de la science, ne sont qu'enfantillages. Ce n'est pas de l'extérieur que nous devons aborder la science, mais par l'intérieur, là où comme le dit Werner Heisenberg : « le chercheur fournit l'effort énorme... de modifier la structure même de sa pensée » (1969, préface à la correspondance Einstein/Born, Le Seuil, 1972). 
LE DISCOBOLE [figure 11]. Parce qu'elle incarne à elle seule la beauté du geste sportif, la sculpture de Myron reste toujours le symbole de l'olympisme. Bien décidé à en renouveler la vision, je postulais l'équivalence entre la double volte de l'athlète et les mouvements circulaires d'un rotor en translation d'après les études de l'ONÉRA, afin d'abolir la frontière entre l'anatomie et les déplacements de l'air. Compte tenu de la dimension temporelle de notre perception du mouvement, elle n'a pas de raison de demeurer étanche. Dans un suprême effort pour s'extraire de la matière fluide qui l'entoure, au-delà d'un simple lancer, c'est de la condition humaine qu'il s'agit.

Mais pour cela il m'a fallu résoudre une somme de difficultés majeures :

1) Mise en perspective des trajectoires principales (mains, pieds, bassin...) d'après des kinogrammes.

2) Dessin des traces laissées dans l'air par ces trajectoires : combinaison de deux rotations et d'une translation, l'athlète traversant les turbulences laissées par le disque.

3) Mise en place du lanceur par des éléments d'anatomie établis comme la conséquence de ces traces : le sens de lecture doit être celui du disque.

4) L'impression de vitesse et de tension maximale étant obtenue par le choix d'arrêter le mouvement juste avant que l'athlète ne lâche l'engin : phase d'accumulation maximale d'énergie, par les décalages dans le temps des axes du bras droit, des épaules et du bassin, avec en référence Rodin et son Homme qui marche. Toute création se nourrissant aussi de l'héritage des grands anciens, la stabilité d'un triangle isocèle central remplace ici les arêtes extérieures des blocs de marbre des Esclaves de Michel-Ange.

5) La perception de cette énergie étant renforcée par les interfaces corps/air déplacé : par endroits (face au torse et au sol) subsistent les vestiges des mouvements antérieurs, dont l'énergie dégradée est exprimée par des contrastes de formes et de matières.

Ce Discobole était destiné à accompagner la candidature de Paris aux Jeux Olympiques de 2012, mais pourquoi pas celle de 2024 ?

Le modèle au tiers a été fondu au domaine de Coubertin à Saint-Rémy lès Chevreuse, ancienne propriété de la nièce de l'initiateur des Jeux Olympiques modernes. Sa réalisation monumentale rappellerait aussi quel fut le rôle du baron Pierre de Coubertin dans la création de l'« université compagnonnique » qu'est devenu l'ensemble de cette fondation.

MÉHARÉES [figures 6 et 15]. Arpentant depuis l'enfance les pentes douces des plages du Cotentin, je m'amusais dès 1996 à y retrouver les phénomènes étudiés à l'ONÉRA, tels que la mer se retirant les suggère par ses sillons dans le sable. Après une prise d'empreinte au plâtre, son interprétation peut commencer lorsque la marée montante me laisse le loisir de la rapporter indemne à l'atelier. Après quelques étapes comparables au travail de médailliste, alternant modelage du positif puis de son moulage négatif, mais rendues plus laborieuses par les surfaces beaucoup plus importantes, la sculpture achevée ne ressemble plus à son modèle marin : elle en propose un relief positif, largement interprété.

En somme, cela ressemble au travail des géologues essayant de reconstituer un animal que sa fossilisation aura transformé en galette. L'animal, c'est l'eau dont ne subsistent que les traces négatives et la galette, c'est le sable. De plus, sachant que les dunes cachent paradoxalement d'immenses réserves d'eau lorsque le sous-sol le permet, déployer parmi elles une scénographie turbulente est d'autant moins absurde qu'elles demeurent elles aussi mouvantes, façonnées par le vent. Parmi ces travaux réalisés sur le sable normand : 
1) Marches au Hasard [figure 6], en plus des franges ordonnées évoquées plus haut, garde aussi en son centre la mémoire d'une allée de Bénard/Kàrmàn, issue de l'épaisseur d'un relief dunaire.

Ces phénomènes sont conjointement étudiés à l'ESPCI dans le laboratoire de Mécanique des Milieux Hétérogènes, réunissant l'étude de la naissance des dunes, l'instabilité hydrodynamique et la turbulence. Preuve s'il en était besoin qu'en remontant à la source au nom de cette quête de l'unité que je partage avec les scientifiques, des formes en apparence sans rapport entre elles sont régies par des lois communes [figures $14 \mathrm{a}, 14 \mathrm{~b}$ ] .

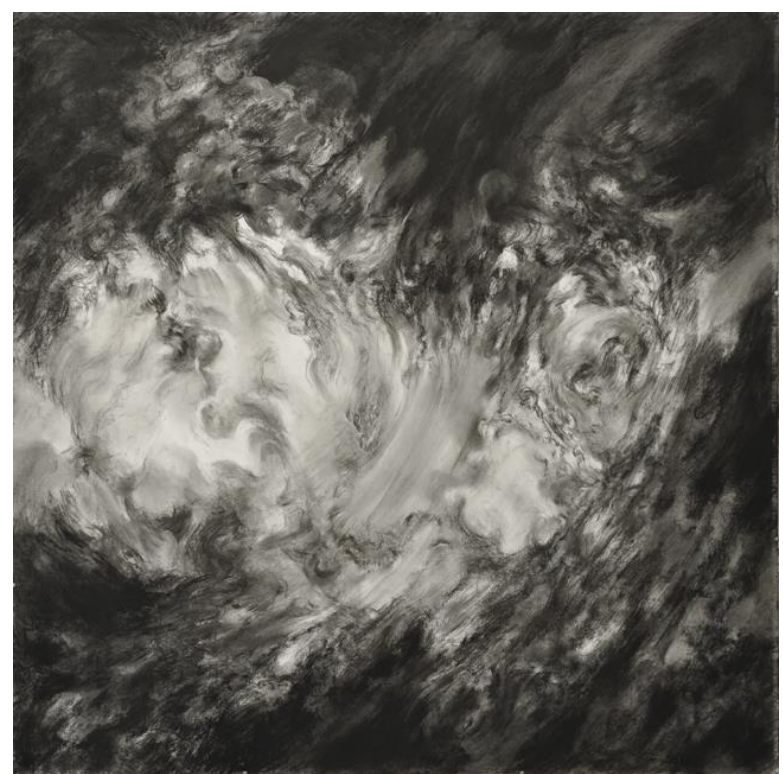

Figure 14a. Nuages - Fusain, 150 × $150 \mathrm{~cm}-2011$

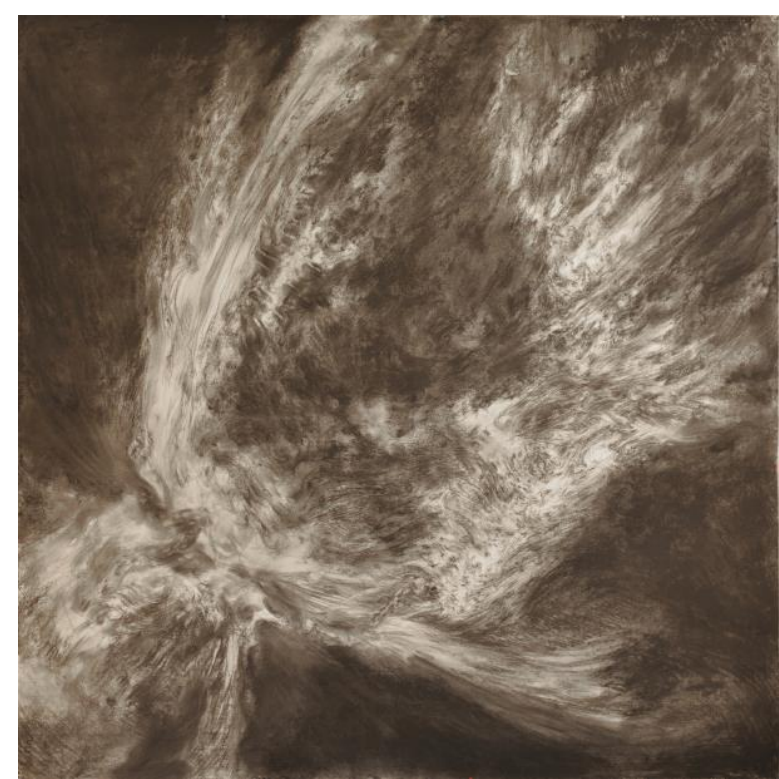

Figure 14b. Nébuleuse -Fusain, 150 × $150 \mathrm{~cm}$ - 2011

2) Table basse [figure 15], issue d'une empreinte de plâtre transposée en marbre et en taille directe, selon une composition en croix de St-André qui n'est pas sans rapport avec un dessin de Léonard que je n'ai découvert que grâce à l'exposition du Louvre (vue figure 16).

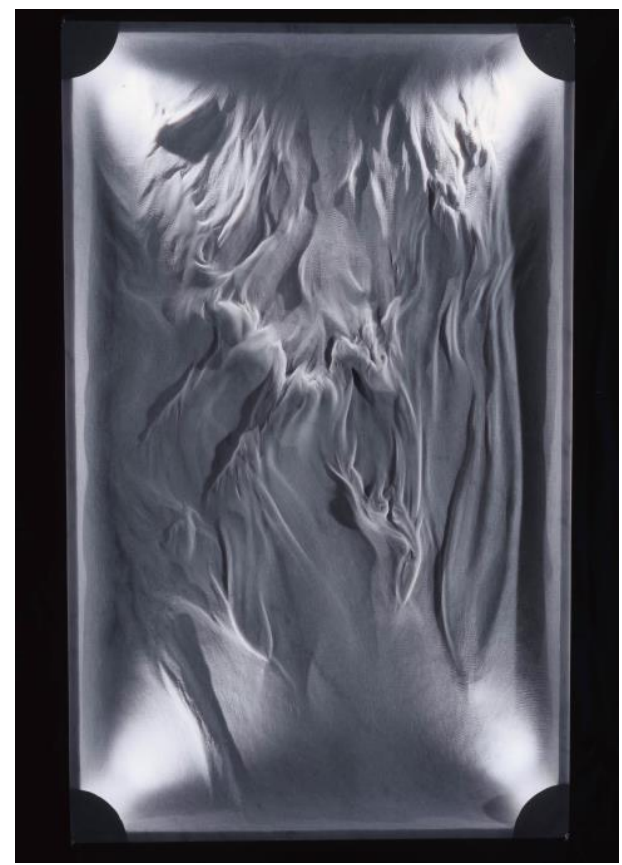

Figure 15. Table basse, marbre $130 \times 80 \times 40 \mathrm{~cm}-2001$

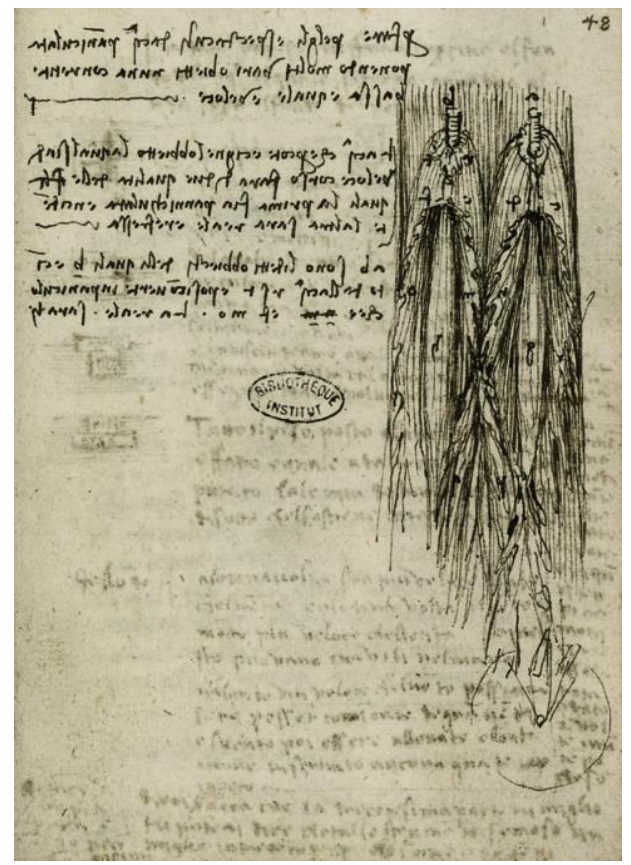

Figure 16. encre bistre - vers 1508/1509,

Paris Institut, $14,6 \times 10,6 \mathrm{~cm}$ 
SAINT-EXUPERY. Encore un «hasard dirigé », mais cette fois témoignage d'une dette de reconnaissance vis-à-vis d'un poète qui fut aussi homme de sciences. L'analyse morphologique qu'il m'a fallu effectuer pour réaliser la médaille du cinquantenaire de sa disparition en 1994 [figure 17] m'a irrésistiblement conduit au portrait de la Stèle de l'aéroport Lyon [figure 18], dialoguant avec son double du Petit Prince suggéré par ces perturbations de la couche limite du profil d'aile qui le soutient, et qu'il avait étudiées avec von Kàrmàn. Double portrait donc, que je réunissais avec le projet Citadelle [figure 7] en une seule et même forme, à l'étude en ce moment avec le Maroc pour un hommage à l'Aéropostale.
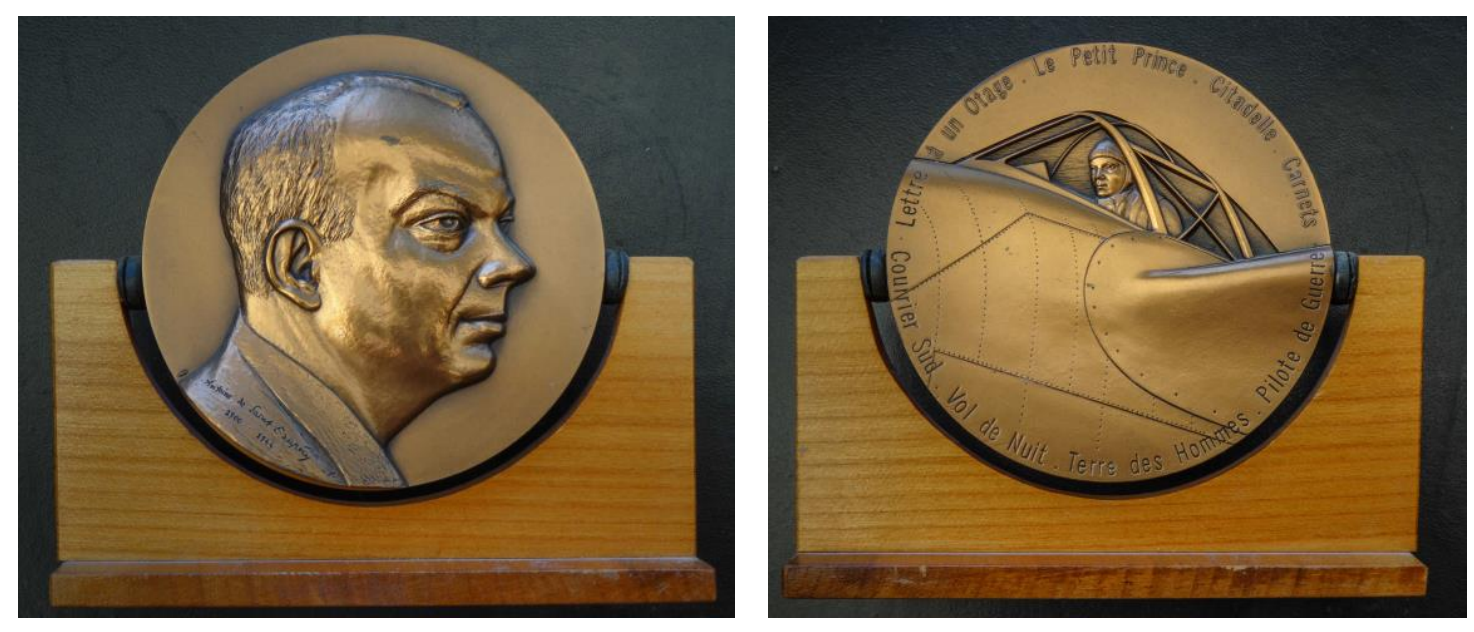

Figure 17. Médaille St-Exupéry - recto/verso, diamètre 8,4 cm -Monnaie de Paris, 1994

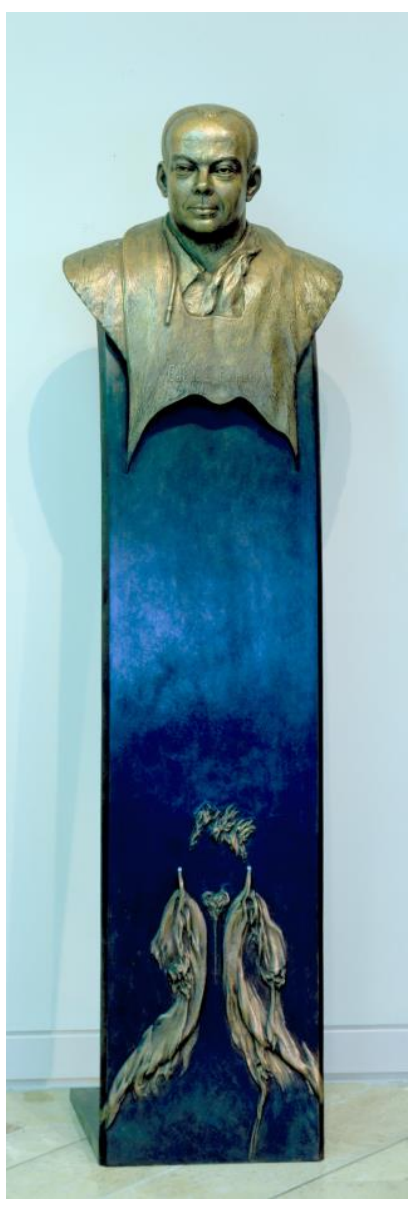

Finalement, qu'il s'agisse de la frontière entre le corps et l'air déplacé, dans la restitution des mouvements de l'eau sur le sable, dans l'étude des nuages [figures 14a, 14b] ou plus directement dans la transposition sculpturale des études de Werlé, tout ceci ne se rapporte qu'à un seul problème: des variations autour du thème de l'énergie, telle que l'observation de la Nature l'ouvre à notre entendement. Les préoccupations humaines meurent avec leur temps, mais comme toute science, les lois de la mécanique des fluides sont éternelles. Et si l'intemporel cher à Malraux ne dépendait que de la part géométrique sous-tendant l'œuvre d'art? $\mathrm{Au}$ delà d'un effet de mode, les rapprochements entre les arts et les sciences trouveraient là leur signification profonde : conférer une part d'éternité à la brièveté de notre existence.

Derniers ouvrages parus :

«Sculpture et Chaos » éditions Connaissances et Savoirs

Préface de Jean-Pierre Luminet, postface de Bruno Chanetz

«Traité de Perspective et Géométrie de la Forme» éditions Eyrolles

Figure 18. Stèle Saint-Exupéry -

bronze, $202 \times 75 \times 75 \mathrm{~cm}-2000$ 